\title{
Wurzelkaries - kennen, erkennen, erfolgreich therapieren
}

Konstantin J. Scholz, Wolfgang Buchalla

\section{Wurzelkaries ist im Kontext des demografischen Wandels eine zunehmende Herausforderung für den Zahnarzt. Dieser Beitrag soll dem Leser nützliche Infor- mationen zu Pathogenese, Prävention und Therapieoptionen an die Hand geben.}

\section{Definition}

Wurzelkaries ist definiert als eine kariöse Läsion im Wurzelzement und -dentin des bleibenden Zahnes apikal der Schmelz-Zement-Grenze [1]. Sie ist häufiger an vestibulären als an lingualen Wurzeloberflächen lokalisiert und insbesondere bei älteren Patienten an Approximalflächen sowie Restaurationsrändern zu finden. Im Gegensatz zu koronal lokalisierten kariösen Läsionen breitet sich Wurzelkaries flächiger und weniger stark in Richtung Pulpa aus [2,3]. Dies kann durch die Pufferwirkung des Sulcusfluids und eine bessere Umspülung mit Speichel im zervikalen Bereich erklärt werden [2,3]. Auf die besonders schwere Form der Karies im Kontext einer Bestrahlung im Kopf-Hals-Bereich soll in diesem Artikel nicht eingegangen werden.

\section{Prävalenz}

Da Wurzelkaries in der Regel nur freiliegende Wurzeloberflächen befällt, nimmt die Prävalenz mit zunehmendem Alter zu. Aufgrund der steigenden Lebenserwartung sowie des längeren Erhalts der Zähne bekommt die Wurzelkaries in Deutschland eine immer größere Bedeutung (DMS V) [4]. In der Altersgruppe der jüngeren Senioren (65-74 Jahre) haben 32\% der Patienten mit Restbezahnung an mindestens einem Zahn Wurzelkaries, wobei männliche Patienten häufiger betroffen sind.

Im Alter von 75 bis 100 Jahren wiesen 16\% der Patienten Wurzelkarieserfahrung auf. Die zahnlosen Menschen dieser Altersgruppe (33\%) sind in alle prozentualen Angaben einbezogen, wodurch die Relevanz der Erkrankung tendenziell unterschätzt wird. Ein RCl (prozentualer Anteil an Wurzeloberflächen mit Karieserfahrung) von 16\% entspricht ca. einem Fünftel aller freiliegenden zervikalen Flächen, wodurch die Wichtigkeit präventiver und restaurativer Maßnahmen bis ins hohe Alter unterstrichen wird [4].
Weitere Faktoren, die ein Auftreten von Wurzelkaries begünstigen, sind unter anderem Parodontitis, schlechte Mundhygiene, bereits aufgetretene Karies im koronalen Bereich, das Tragen von Teilprothesen sowie Alkoholund Tabakkonsum [5].

\section{Pathogenese}

Durch Gingivarezession oder Zahnwanderung können approximal unter sich gehende Bereiche entstehen, die eine Plaqueretention begünstigen, was häufig bei älteren Patienten beobachtet werden kann [6].

Auch bei Wurzelkaries ist die Häufigkeit saccharosereicher Nahrung ein entscheidender ätiologischer Faktor [7]. Klebrige, weiche Kost ohne mechanischen Reinigungseffekt bei der Mastikation kann die Entstehung kariöser Läsionen begünstigen. Unter dem dentalen Biofilm ist das Kollagen freiliegender Wurzeloberflächen $\mathrm{pH}$ abhängigen bakteriellen Proteasen ausgesetzt, was zu einer Degradation des Kollagens führen und den Kariesprozess beschleunigen kann [8].

Die Entstehung von Karies an Wurzeloberflächen geht häufig mit eingeschränkten Speichelfluss einher [9, 10]. Der Speichel unterstützt die Clearance von Nahrungsbestandteilen aus der Mundhöhle, hat Pufferfunktion und ist durch die Übersättigung mit Kalzium und Phosphat kariesprotektiv. Verminderter Speichelfluss (Oligosialie), häufig auftretend mit einer subjektiv empfundenen Mundtrockenheit (Xerostomie), sind Nebenwirkungen von Medikamenten und treten daher besonders bei älteren oder multimorbiden Patienten auf [11]. Sulcusfluid weist zwar eine Pufferfunktion auf, enthält aufgrund seiner serumähnlichen Zusammensetzung aber essenzielle Nährstoffe für pathogene Bakterien (Eisen, Proteine) und kann auf diese Weise kariöse Prozesse beschleunigen [12]. 


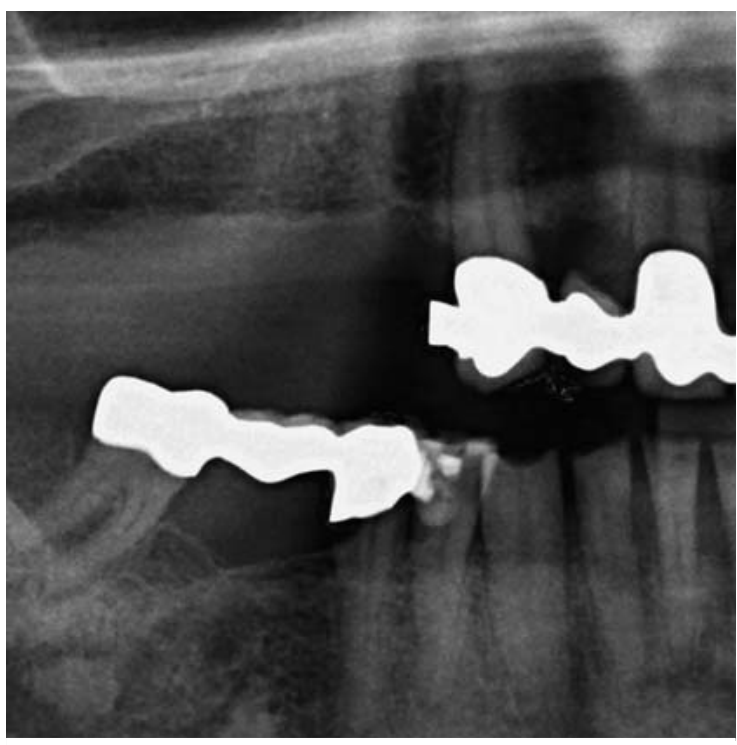

- Abb. 1 Dieses Orthopantomogramm (Ausschnitt 1. und 4. Quadrant) verdeutlicht die Problematik röntgenologischer Diagnostik im zervikalen Bereich. Während die Karies am distalen Restaurationsrand von 45 deutlich erkennbar war und sich auch klinisch gezeigt hat, konnte der Verdacht einer weiteren Läsion an 44 distal klinisch nicht bestätigt werden.

\section{Diagnostik}

Die vestibulären und oralen kariösen Läsionen sind zur klinischen Inspektion und taktilen Untersuchung in der Regel gut zugänglich. Erschwert wird die Einsicht bei subgingivaler Lokalisation und subgingivalen Restaurationsrändern. In 2-dimensionalen Röntgenaufnahmen (Zahnfilm, OPG) sind vestibuläre und orale Wurzelkaries nicht zuverlässig erkennbar.

In approximalen Bereichen dagegen sind Einzelzahn- und Panoramaschichtaufnahmen eine sinnvolle Ergänzung zur Detektion kariöser Läsionen im Wurzel- und Restaurationsbereich im Vergleich zu alleiniger klinischer Inspektion [13]. Aufhellungen im approximal-zervikalen Bereich werden aufgrund zweier Effekte jedoch häufig überinterpretiert ( $\bullet$ Abb. 1). In Bereichen benachbarter heller und dunkler Areale werden im menschlichen Auge die Kontraste verstärkt (Mach-Band-Effekt) [14]. Bei zahnärztlichen Röntgenbildern ist dies häufig der Fall, wenn im zervikalen Bereich eine dünne Schmelzschicht, der Alveolarknochen sowie ein nicht vom Alveolarknochen gefasster Dentinbereich zusammentreffen. Dieser „Burnout-Effekt“" wird durch die interdental geringere Dicke der Zahnhartsubstanz noch verstärkt $[15,16]$.

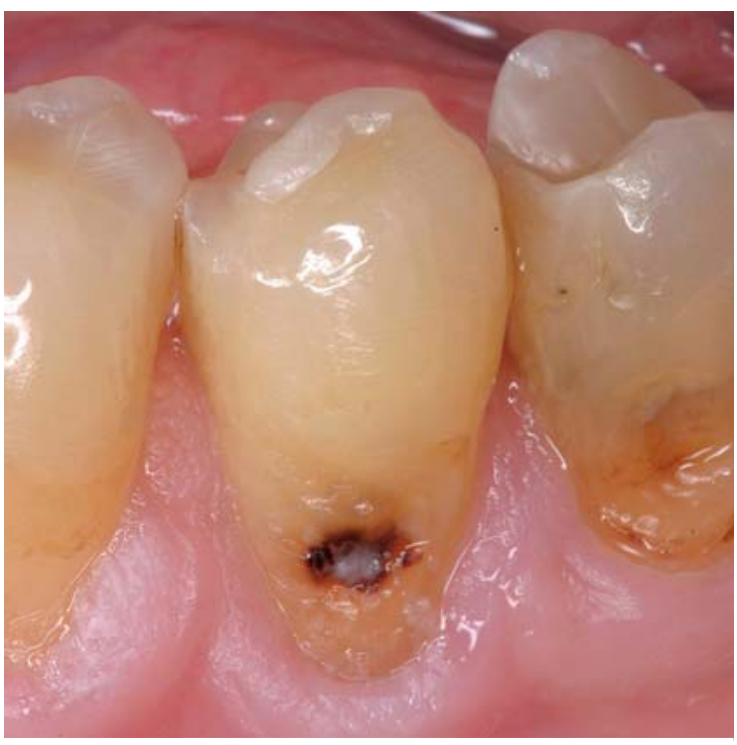

- Abb. 2 Beginnende Wurzelkaries ohne Kavitation. Biofilm (Plaque) als Ursache der Wurzelkaries ist deutlich zu erkennen. Läsionen dieser Art können durch eine verbesserte Mundhygiene und weniger kariogene Ernährung erfolgreich noninvasiv therapiert werden.

Merke

Aufgrund des Burnout- und Mach-Band-Effekts sollte der Verdacht auf approximal-zervikale Karies vor der Therapie immer durch die klinische Untersuchung und gründliche, aber vorsichtige Sondierung bestätigt werden $[17,18]$.

Lichtbasierte Alternativen zur klinischen und radiologischen Diagnostik sind aufgrund der erschwerten Zugänglichkeit und Sicht für die Detektion der Wurzelkaries im approximalen Bereich nur stark eingeschränkt anwendbar. Die Anwendung der Laserfluoreszenz (Diagnodent) in zugänglichen Bereichen wurde vorgeschlagen, ist aber mit großen Unsicherheiten behaftet $[19,20]$. Gleiches gilt für Methoden auf Basis der elektrischen Widerstandsmessung.

Die vorsichtige, aber gründliche Sondierung mit feinen gebogenen Sonden ist zur Erkennung der approximalen und interradikulären Wurzelkaries, gerade auch an Rändern von Restaurationen und Kronen, das Mittel der Wahl und sollte insbesondere bei älteren und Risikopatienten regelmäßig Anwendung finden.

\section{Läsionsaktivität}

Im Hinblick auf die Therapieentscheidung kann die Kenntnis über die Aktivität der Wurzelkaries von Bedeutung sein. Aktive, voranschreitende Läsionen weisen eine bei Sondierung erweichte, lederartige Oberfläche auf. In- 
aktive Läsionen haben eine harte, oft sehr dunkel verfärbte Oberfläche und breiten sich nicht weiter aus. Inaktive kariöse Läsionen im Wurzelbereich bedürfen im Grunde keiner Veränderung der aktuellen präventiven Maßnahmen und Ernährungsgewohnheiten. Aktive Läsionen dagegen können häufig durch eine Intensivierung der Mundhygiene und Verbesserung der Ernährung inaktiviert werden [21].

\section{Prävention und noninvasive Therapie}

Die Empfehlungen zur Prävention kariöser Läsionen an Wurzeloberflächen decken sich weitgehend mit Läsionen des koronalen Bereichs. Neben einer suffizienten Mundhygiene und Biofilmentfernung, Applikation lokaler Kariostatika und regelmäßiger zahnärztlicher Inspektion ist eine mundgesunde Ernährung von Bedeutung. Entscheidend ist die tägliche, gründliche Zahnreinigung mit einer fluoridhaltigen Zahnpasta und eine Reduktion der Menge und insbesondere Frequenz kariogener Mahlzeiten [22]. Vor allem bei älteren Patienten kann die Reinigung der Approximalräume eine besondere Herausforderung darstellen, für die eine individuelle Lösung erarbeitet und eingeübt werden muss.

Die Therapie kariöser Läsionen im Wurzelbereich bedeutet nicht zwangsläufig, dass kariös veränderte Anteile exkaviert und der Defekt restauriert werden muss. Gerade gut erreichbare Läsionen ohne Einbruch der Oberfläche können mit geeigneten noninvasiven Maßnahmen sehr erfolgreich von einem „aktiven“ in einen „arretierten“ Zustand überführt werden ( $\bullet$ Abb. 2). Dies kann jedoch nur gelingen, wenn sich die lokalen, bisher kariogenen Bedingungen an der betroffenen Wurzeloberfläche durch Verhaltensänderung des Patienten verbessern. Das gleiche gilt auch für restaurativ therapierte Läsionen, da nur mit der Beseitigung der Ursachen das Entstehen neuer kariöser Läsionen an den der Restauration angrenzenden Bereichen verhindert werden kann ( $\mathbf{A} \mathbf{A b} \mathbf{b} . \mathbf{3})$.

Wurzelkaries zeichnet sich häufig durch eine intakte, hypermineralisierte Schicht mit darunterliegendem Läsionskörper aus. Aufgrund der geringeren Größe und weniger dichten Anordnung von Apatitkristallen im Vergleich zu Schmelz können Mineralien im Remineralisationsprozess besser ins Hartgewebe des Wurzeldentins diffundieren [23]. Durch konsequente Biofilmentfernung und die Anwendung einer fluoridhaltigen Zahnpasta können aktive, klinisch weich-ledrige Läsionen in inaktive, sondenharte Läsionen überführt werden ( $\bullet$ Abb. $\mathbf{4 a}$ bis $\mathbf{c}$ ). Dabei kann eine fluoridhaltige Zahnpasta mit erhöhtem Fluoridgehalt bis 5000 ppm Fluoridgehalt sinnvoll sein [23].

Silber-Diamin-Fluorid, in Deutschland nicht als Präparat erhältlich, gilt ebenfalls als erfolgversprechende Therapieoption zur Arretierung gut zugänglicher kariöser Läsionen, wobei die starke Schwarzfärbungen der behan- 


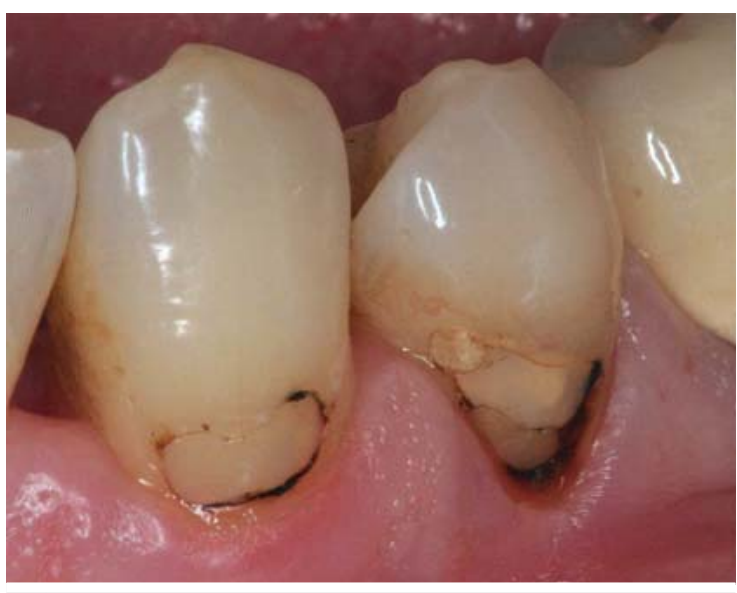

- Abb. 3 Sequenzielle Füllungstherapie ohne präventive Maßnahmen. Die Füllungstherapie von Wurzelkaries kann nur erfolgreich sein, wenn die lokale Mundhygiene verbessert wird.

delten Flächen die Anwendung sehr stark einschränkt $[24,25]$. Eine Empfehlung zu neueren Kariostatika wie Hydroxylapatitpasten oder CPP-ACP im zervikalen Bereich ist aufgrund der bislang unzureichenden klinischen Studienlage nicht möglich. Insbesondere bei noch vorhandenem Speichelfluss kann aber von einem eher eingeschränkten Zusatznutzen Kalzium-Phosphat-basierter Präparate ausgegangen werden. Hier zeigen Fluoride eine bessere Effektivität.

Zur Therapie verminderten Speichelflusses und Prävention dadurch begünstigter kariöser Läsionen können zusätzlich zur Anwendung von Fluoriden Speichelstimulanzien wie Pilocarpin eingesetzt werden. Als Basismaßnahme kann in den meisten Fällen das Kauen zuckerfreier Kaugummis empfohlen werden [9]. Um kariöse Prozesse nicht zusätzlich zu beschleunigen, ist bei Anwendung von feuchtigkeitsspendenden Gelen, Sprays, Spülungen und Speichelersatzprodukten auf einen $\mathrm{pH}$-Wert von mindestens 6,7 zu achten, der von einigen Produkten deutlich unterschritten wird [26].

\section{Cave}

Beim Einsatz von Sialogoga und Speichelersatzmedien gilt es, deren kariogenes und erosives Potenzial gegenüber dem therapeutischen Nutzen streng abzuwiegen.

Bei älteren Patienten ist die manuelle Geschicklichkeit oft eingeschränkt [27]. Besonders bei Patienten in Pflegeeinrichtungen ist die Unterstützung des Pflegepersonals bei der Zahn- und Prothesenpflege sowie der Ernährung für die orale und allgemeine Gesundheit essenziell $[28,29]$.
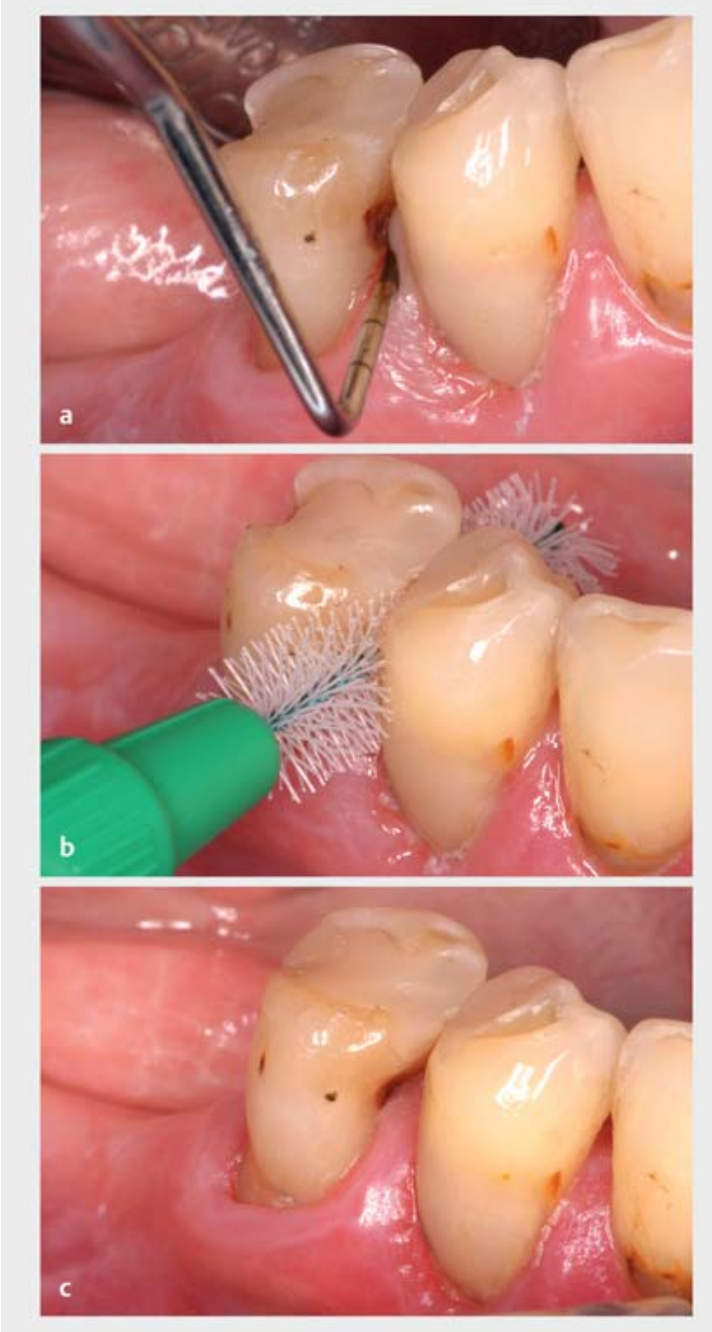

- Abb.4 Approximal gelegene Wurzelkaries bei einer 71-jährigen Patientin. a Leicht eingebrochene, erweichte Oberfläche. b Noninvasive Therapie mit Instruktion zur Interdentalraumhygiene und Fluoridierung. c Nach 5 Monaten zeigt sich eine arretierte kariöse Läsion mit harter Oberfläche bei etwas stärkerer Verfärbung.

\section{Invasive und restaurative Therapie}

\section{Merke}

Da der gingivale Rand von Restaurationen die Entstehung von Wurzelkaries begünstigen kann, trägt eine möglichst koronale Positionierung des Restaurationsrandes zur Prävention der Wurzelkaries bei [30].

Eine invasive Füllungstherapie bei kariösen Läsionen im zervikalen Bereich sollte erst durchgeführt werden, wenn noninvasive Maßnahmen nicht ausreichend waren oder nicht erfolgversprechend sind. Dies ist insbesondere dann der Fall, wenn die Läsionen für den Patienten aufgrund der Lokalisation oder eines Einbruchs der Oberfläche nicht zu reinigen sind [23]. Bei größeren zervikalen 

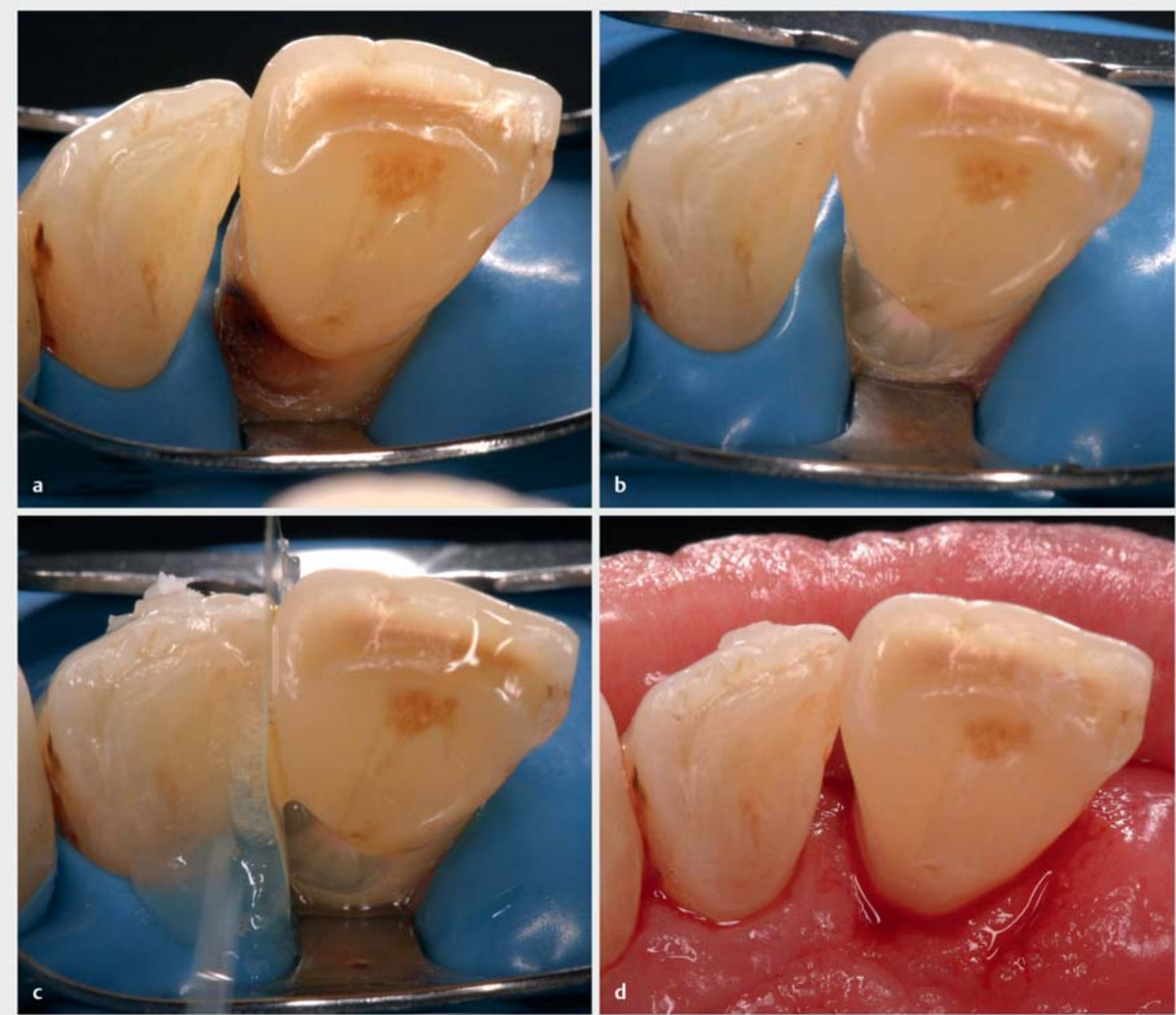

- Abb. 5 Tief subgingivale Wurzelkaries bei einer 70-jährigen Patientin. a Die Gingivaretraktion mit Kofferdam und tief inserierter Frontzahnklammer zeigt die gesamte Ausdehnung der Wurzelkaries. b Situation nach Kariesexkavation. c Am Nachbarzahn mit Adhäsiv verklebte Matrize. $\mathbf{d}$ Situation nach Fertigstellung mit einem Hybridkomposit.

kariösen Defekten ist eine Füllungstherapie in der Regel sinnvoll.

Bei der Kariesexkavation ist auf eine vollständige Entfernung kariös erweichten Dentins im Bereich der Präparationsgrenze zu achten, um bei adhäsiver Versorgung einen möglichst optimalen Haftverbund zwischen Adhäsiv und Dentin sicherzustellen. Bei einer pulpanahen Wurzelkaries ist bei ansonsten symptomlosen, vitalen Zähnen eine selektive Kariesexkavation im pulpanahen Bereich häufig erfolgreich [31].

Für die restaurative Therapie der Wurzelkaries kommen in der Regel plastische Restaurationen, seltener Kronen infrage. Kronen haben immer den Nachteil, dass aufgrund des gegenüber dem Zahnäquator kleineren Wurzelquerschnitts große koronale Anteile bei der Präparation entfernt werden müssen, wenn die Kronenränder unterhalb des Zahnhalses zu liegen kommen [32,33]. Bei vitalen Zähnen erhöht dies zudem die Gefahr der Pulpaeröffnung oder der Pulpanekrose durch ein Präparationstrauma.

Aufgrund der gingivalen oder subgingivalen Lage des Präparationsrandes ist die Gingivaretraktion entscheidend, um Füllungswerkstoffe adhäsiv verarbeiten zu können. Bei approximaler Lage der Läsion wird mit einem Zugang von oral oder vestibulär weniger gesunde Zahnhartsubstanz entfernt als von okklusal. Eine retentive Präparation ist mit den heute verwendeten Werkstoffen nicht mehr sinnvoll. Dagegen ist die Verwendung von Kofferdam grundsätzlich zu empfehlen ( $\bullet$ Abb.5a bis d). Approximal führt er meist zu einer guten Retraktion und dem Schutz der Gingiva. Bukkal ist die Anwendung manchmal nur eingeschränkt möglich. Hier ist neben den üblichen Maßnahmen der relativen Trockenlegung die Anwendung von in Aluminiumchlorid getränkten, geflochtenen Re- 

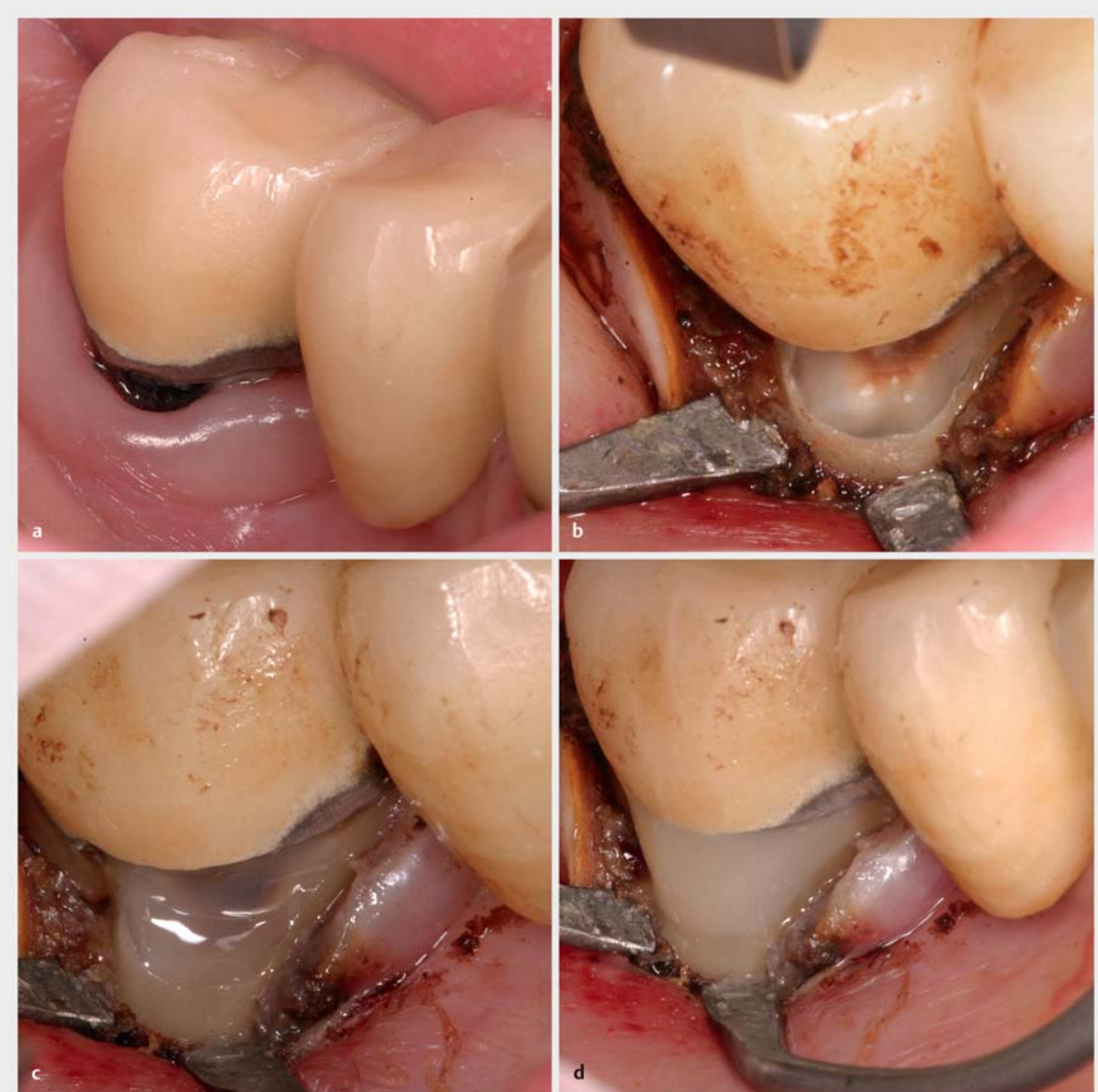

- Abb. 6 Reparaturfüllung an einer Wurzelkaries am Brückenpfeiler bei einer 68-jährigen Patientin. a Subgingivale Ausdehnung der Kronenrandkaries. b Kariesexkavation und Präparation nach Gingivektomie, Blutstillung mit Eisen-III-Sulfat und Gingivaretraktion. c Situation nach Applikation des Adhäsivs und einer Schicht Flow-Komposit. d Situation nach Applikation der letzten Schicht Flow-Komposit. Die Vermeidung von Überschüssen erleichtert die spätere Ausarbeitung.

traktionsfäden nützlich, wobei die Applikation der Fäden bereits vor der Präparation sinnvoll ist. Retraktionsfäden haben ähnlich wie Kofferdam den Zusatznutzen, dass der Ausstrom von Sulcusflüssigkeit reduziert wird [12,34].

Bei Defekten, die tief unter die Gingiva reichen, ist eine Verletzung nicht immer auszuschließen. Die Gingivektomie mit dem Elektrotom ist eine weitere Möglichkeit, den Zugang zu subgingivalen kariösen Läsionen zu gewährleisten. Für die Indikationsstellung sollte aber immer der zu erwartende Gingivaverlauf nach der Therapie bedacht werden, da der Verlust an Gingiva z. B. im appro- ximalen Bereich auch zu erschwerter Reinigung und höherer Speiseimpaktion beitragen kann [6].

Eine weitere Herausforderung für die restaurative Therapie ist die Wurzelkaries im Bereich von Kronenrändern, insbesondere bei unterminierender Ausbreitung. Vor allem bei älteren Patienten ist im approximalen Bereich mit Wurzelkaries am Kronenrand zu rechnen. In der Gerodontologie ist es oft sinnvoll, eine Neuanfertigung der gesamten Krone zu vermeiden, insbesondere, wenn die Krone Teil eines Brückenverbandes ist ( $\bullet \mathbf{A b b} \mathbf{6} \mathbf{6}$ bis $\mathbf{d}$ ). 

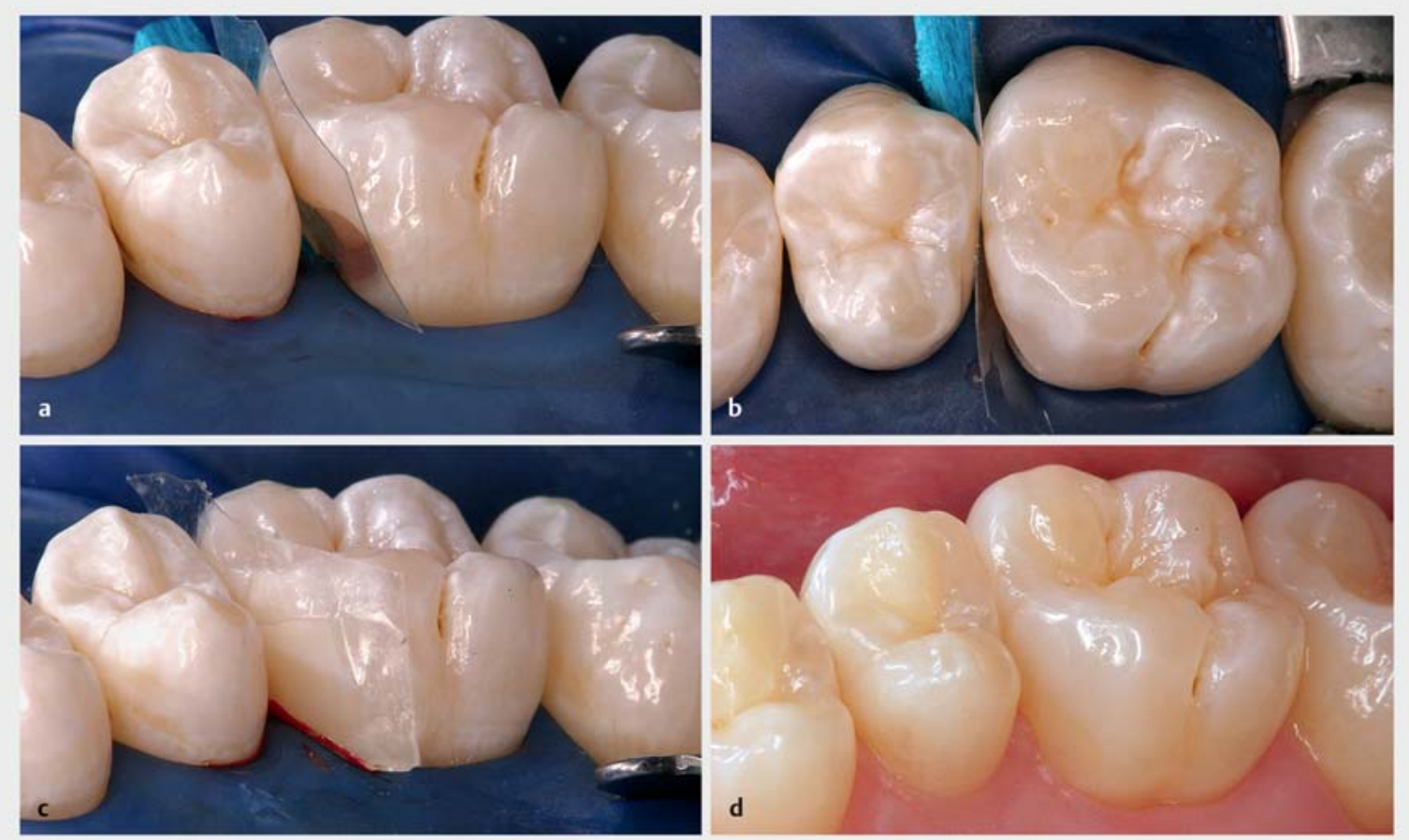

- Abb. 7 Restauration tiefliegender approximaler Läsionen mit Matrizenband und Flow-Komposit. a, b Das Matrizenband ist einseitig verkeilt und wird zusätzlich vom Kofferdam adaptiert. c Kavität mit Flow-Komposit ausgespritzt. d Restauration 2 Jahre nach Fertigstellung.

\section{Restaurationsmaterialien und Adhäsivsysteme}

Für die direkte restaurative Therapie mit plastischen Materialien kommen nur adhäsive Verfahren, insbesondere Kompositfüllungen, polyalkensäuremodifizierte Komposite, konventionelle oder resinmodifizierte Glasionomerzemente sowie selbstadhäsive Komposite infrage. Die Auswahl des geeigneten Verfahrens richtet sich immer nach der individuellen Situation, insbesondere Sicht und Zugänglichkeit, Möglichkeiten der Trockenlegung und Prognose des Zahnes.

Merke

Je komplexer und anspruchsvoller die Versorgung einer kariösen Läsion im Wurzelbereich ist, desto einfacher zu verarbeiten sollte das gewählte Restaurationssystem sein.

Wenn die zur Aufnahme des Füllungsmaterials vorbereitete Kavität ausreichend lange trocken gehalten werden kann, sind adhäsiv verankerte Komposite das Material der Wahl. Insbesondere bei gemischten Substraten, z. B. am Restaurationsrand, bieten Adhäsivsysteme mit sauren Phosphatmonomeren (z. B. MDP) eine zusätzliche Adhäsion an Gold- und Keramikoberflächen [35]. Ist ein ausreichend langes Trockenhalten nicht gewährleistet, können konventionelle oder resinmodifizierte Glasionomerzemente trotz mechanischer Nachteile vorteilhaft sein [36]. Approximale Defekte lassen sich nach Einlegen einer Teilmatrize vor Feuchtigkeitszutritt schützen und dann, nach Adhäsivapplikation, mit einem Flow-Komposit „ausspritzen" ( $\triangleright$ Abb. 7a bis d).

Bei einer primären zervikalen Restauration werden durch selektive Schmelzätzung in Kombination mit Self-EtchAdhäsiven gute Ergebnisse erreicht [37]. Bei der Therapie zervikaler Sekundärkaries an Restaurationen auf Metalloder Metalloxidbasis führt die Kontamination der Restaurationsmaterialien mit Phosphorsäure zu einem schlechteren Haftverbund [38]. Aufgrund der unterschiedlichen denkbaren Substrate im zervikalen Bereich stellen Universaladhäsive einen vielversprechenden Ansatz dar, der durch Abstrahlen mit $\mathrm{Al}_{2} \mathrm{O}_{3}(27 \mu \mathrm{m})$ von Komposit-, Metall- und Keramikanteilen weiter optimiert werden kann.

Eine aktuelle Metaanalyse verglich Restaurationen zervikaler Läsionen aus konventionellem und resinmodifiziertem Glasionomerzement mit Kompositen [39]. Glasionomerzemente zeigten etwas bessere Retentionsraten, jedoch auch schlechtere Farbadaptation und höhere Oberflächenrauigkeit, die relevant für die Akkumulation von Biofilm sein kann. In den Kriterien Sekundärkaries und marginale Adaptation gab es keine Unterschiede [39]. 
Bei der Therapie von kavitierten Läsionen an Restaurationsrändern gibt es bislang keine Richtlinien, wann Restaurationen vollständig abgenommen und erneuert werden müssen und bis wann eine minimalinvasive Reparaturfüllung ohne Entfernen der Restauration möglich ist [40].

\section{FAZIT}

Aufgrund der steigenden Prävalenz von Wurzelkaries in einer älter werdenden Bevölkerung und der oft schwierigen Therapierbarkeit stehen eine ausführliche und regelmäßige Befundung und präventive Maßnahmen im Vordergrund. Gut zugängliche Läsionen lassen sich oft noninvasiv therapieren. Die Art der restaurativen Therapie richtet sich stark nach den individuellen und lokalen Gegebenheiten, wobei Trockenlegung, Gingivaretraktion und, insbesondere bei approximaler Lage, der Zugang wesentliche Entscheidungskriterien darstellen. Grundsätzlich sollten nach einer restaurativen Therapie die gleichen präventiven Maßnahmen (Reinigung und Fluoridierung) implementiert werden wie bei einer noninvasiven Therapie.

\section{Interessenkonflikt}

Die Autoren geben an, dass kein Interessenkonflikt besteht.

\section{Autorinnen/Autoren}

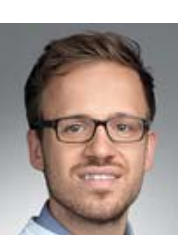

\section{Konstantin J. Scholz}

Dr. med. dent., Poliklinik für Zahnerhaltung und Parodontologie, Universitätsklinikum Regensburg

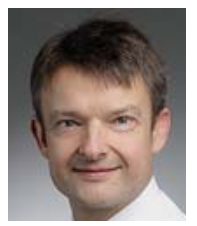

\section{Wolfgang Buchalla}

Prof. Dr. med. dent., Poliklinik für Zahnerhaltung und Parodontologie, Universitätsklinikum Regensburg

\section{Korrespondenzadresse}

Dr. med. dent. Konstantin J. Scholz

Poliklinik für Zahnerhaltung und Parodontologie Universitätsklinikum Regensburg

Franz-Josef-Strauß-Allee 11

93053 Regensburg

\section{Literatur}

[1] Sumney DL, Jordan HV, Englander HR. The Prevalence of Root Surface Caries in Selected Populations. J Periodontol 1973; 44: 500-504

[2] Takahashi N, Nyvad B. Ecological Hypothesis of Dentin and Root Caries. Caries Res 2016; 50: 422-431

[3] Damé-Teixeira N, Parolo CCF, Maltz M. Specificities of Caries on Root Surface. Monogr Oral Sci 2017; 26: 15-25

[4] Jordan AR, Micheelis W. Fünfte Deutsche Mundgesundheitsstudie (DMS V). Köln: Deutscher Zahnärzteverlag; 2016

[5] Hayes M, Burke F, Allen PF. Etiology, Risk Factors and Groups of Risk. Monogr Oral Sci 2017; 26: 9-14

[6] Henriques PG, Okajima LS, Siqueira S. Surgical reconstruction of the interdental papilla: 2 case reports. Gen Dent 2018; 66: e1-e4

[7] Papas AS, Joshi A, Palmer CA et al. Relationship of Diet to Root Caries. Am J Clin Nutr 1995; 61: 423S-429S

[8] Tjäderhane L, Buzalaf MAR, Carrilho M et al. Matrix Metalloproteinases and Other Matrix Proteinases in Relation to Cariology: The Era of 'Dentin Degradomics'. Caries Res 2015; 49: 193-208

[9] Plemons JM, Al-Hashimi I, Marek CL. Managing xerostomia and salivary gland hypofunction: Executive summary of a report from the American Dental Association Council on Scientific Affairs. J Am Dent Assoc 2014; 145: 867-873

[10] Papas AS, Joshi A, MacDonald SL et al. Caries prevalence in xerostomic individuals. J Can Dent Assoc 1993; 59: 171-174, 177-179

[11] Navazesh M, Kumar SKS. Xerostomia: prevalence, diagnosis, and management. Compend Contin Educ Dent 2009; 30: 326-324

[12] Do T, Damé-Teixeira N, Naginyte M et al. Root Surface Biofilms and Caries. Monogr Oral Sci 2017; 26: 26-34

[13] Kweon HH-I, Lee J-H, Youk T-M et al. Panoramic radiography can be an effective diagnostic tool adjunctive to oral examinations in the national health checkup program. J Periodontal Implant Sci 2018; 48: 317-325

[14] Lane E], Proto AV, Phillips TW. Mach Bands and Density Perception. Radiology 1976; 121: 9-17

[15] Berry HM jr. Cervical Burnout and Mach Band: Two Shadows of Doubt in Radiologic Interpretation of Carious Lesions. J Am Dent Assoc 1983; 106: 622-625

[16] Pasler FA. Zahnärztliche Radiologie. 5. Aufl. Stuttgart: Thieme; 2017

[17] Wittneben J-G, Zöllner A, Wright AF et al. Comparison of visual-tactile, radiographic, and histologic diagnoses of subgingival crown margin caries- an in vitro study. Int J Prosthodont 2009; 22: 561-565

[18] Zoellner A, Heuermann M, Weber H-P et al. Secondary caries in crowned teeth: correlation of clinical and radiographic findings. J Prosthet Dent 2002; 88: 314-319

[19] Haak R, Wicht MJ. Caries detection and quantification with DIAGNOdent: prospects for occlusal and root caries? Int J Comput Dent 2004; 7: 347-358

[20] Pretty IA. Monitoring of Root Caries Lesions. Monogr Oral Sci 2017; 26: 70-75

[21] Carvalho TS, Lussi A. Assessment of Root Caries Lesion Activity and Its Histopathological Features. Monogr Oral Sci 2017; 26: 63-69 
[22] Rodrigues JA, Lussi A, Seemann R et al. Prevention of crown and root caries in adults. Periodontol 2000 2011; 55: 231-249

[23] Heasman PA, Ritchie M, Asuni A et al. Gingival recession and root caries in the ageing population: a critical evaluation of treatments. J Clin Periodontol 2017; 44: S178-S193

[24] Wierichs RJ, Meyer-Lueckel H. Systematic Review on Noninvasive Treatment of Root Caries Lesions. J Dent Res 2015; 94: 261-271

[25] Seifo N, Cassie H, Radford JR et al. Silver diamine fluoride for managing carious lesions: an umbrella review. BMC Oral Health 2019; 19: 145. doi:10.1186/s12903-019-0830-5

[26] Delgado AJ, Olafsson VG. Acidic oral moisturizers with $\mathrm{pH}$ below 6.7 may be harmful to teeth depending on formulation: a short report. Clin Cosmet Investig Dent 2017; 9: 81-83

[27] Grönbeck Lindén I, Hägglin C, Gahnberg L et al. Factors Affecting Older Persons' Ability to Manage Oral Hygiene: A Qualitative Study. JDR Clin Trans Res 2017; 2: 223-232

[28] Jennings A. Assessing Oral Hygiene in Hospitalized Older Veterans. Medsurg Nurs 2015; 24: 420-424

[29] Coker E, Ploeg J, Kaasalainen S et al. Observations of oral hygiene care interventions provided by nurses to hospitalized older people. Geriatr Nurs 2017; 38: 17-21

[30] Mjör IA. The location of clinically diagnosed secondary caries. Quintessence Int 1998; 29: 313-317

[31] Buchalla W, Frankenberger R, Galler K et al. Aktuelle Empfehlungen zur Kariesexkavation. Wissenschaftliche Mitteilung der Deutschen Gesellschaft für Zahnerhaltung (DGZ). Dtsch Zahnarztl Z 2017; 72: 484-494

[32] Edelhoff D, Sorensen JA. Tooth structure removal associated with various preparation designs for anterior teeth. J Prosthet Dent 2002; 87: 503-509
[33] Edelhoff D, Sorensen JA. Tooth structure removal associated with various preparation designs for posterior teeth. Int J Periodontics Restorative Dent 2002; 22: 241-249

[34] Tabassum S, Adnan S, Khan FR. Gingival Retraction Methods: A Systematic Review. J Prosthodont 2017; 26: 637-643

[35] Kanzow P, Wiegand A, Schwendicke F et al. Same, same, but different? A systematic review of protocols for restoration repair. J Dent 2019; 86: 1-16

[36] Celik EU, Tunac AT, Yilmaz F. A Randomized, Controlled, Splitmouth Trial Evaluating the Clinical Performance of High-viscosity Glass-ionomer Restorations in Noncarious Cervical Lesions: Two-year Results. J Adhes Dent 2018; 20: 299-305

[37] Szesz A, Parreiras S, Reis A et al. Selective enamel etching in cervical lesions for self-etch adhesives: A systematic review and meta-analysis. J Dent 2016; 53: 1-11

[38] Göstemeyer G, Blunck U. Reparatur/Korrektur von Restaurationen. ZWR 2016; 125: 167-168

[39] Boing TF, de Geus JL, Wambier LM et al. Are Glass-Ionomer Cement Restorations in Cervical Lesions More Long-Lasting than Resin-based Composite Resins? A Systematic Review and Meta-Analysis. J Adhes Dent 2018; 20: 435-452

[40] Alomari Q, Al-Saiegh F, Qudeimat M et al. Recurrent caries at crown margins: making a decision on treatment. Med Princ Pract 2009; 18: 187-192

Bibliografie

DOI https://doi.org/10.1055/a-1011-7974

ZWR - Das Deutsche Zahnärzteblatt 2019; 128: 501-509

(c) Georg Thieme Verlag KG Stuttgart · New York ISSN 0044-166X 